\title{
Magnetic susceptibility and specific heat of the Anderson lattice: Perturbative expansion around the atomic limit
}

\author{
L. G. Brunet, M. A. Gusmão, and J. R. Iglesias \\ Instituto de Física, Universidade Federal do Rio Grande do Sul, Caixa Postal 15051, 91501 Porto Alegre, \\ Rio Grande do Sul, Brazil \\ (Received 8 November 1991)
}

\begin{abstract}
The Green's functions relevant to the periodic Anderson Hamiltonian are calculated via perturbation theory around the atomic limit. The approximation reproduces exact results in three different limits: zero bandwidth, zero hybridization, and zero Coulomb correlation. The density of states, the magnetic susceptibility, and the electronic specific heat are calculated and discussed in both the Kondo and intermediate-valence regimes for different values of hybridization and Coulomb repulsion. The results are in qualitative agreement with experiments and are related to other theoretical calculations.
\end{abstract}

\section{INTRODUCTION}

A large class of rare-earth and actinide compounds exhibit anomalous properties that vary from intermediate valence (IV) (such as SmS) to Kondo lattices and heavyfermion systems (such as $\mathrm{CeCu}_{2} \mathrm{Si}_{2}$ ). ${ }^{1}$ In these compounds the linear coefficient $(\gamma)$ of the electronic specific heat increases from the usual value of $4-10 \mathrm{~mJ} / \mathrm{mol} \mathrm{K}^{2}$ for normal metals, reaching $10^{2} \mathrm{~mJ} / \mathrm{mol} \mathrm{K}$ for IV compounds and $10^{3} \mathrm{~mJ} / \mathrm{mol} \mathrm{K}^{2}$ for heavy-fermion systems. ${ }^{2}$ Although specific differences may be stated between IV and Kondo systems, common characteristics can be found, such as decreasing values for the static susceptibility at high temperatures, Pauli-like susceptibility at low temperatures-in the nonmagnetic compounds-and one or more peaks in the specific heat, indicating high electronic effective masses.

A suitable description of rare-earth Kondo and IV systems should take into account the following characteristics: (a) strongly correlated $f$ electrons; (b) delocalized $(5 d, 6 s)$ conduction electrons; (c) mixing between $f$ and conduction $(c)$ electrons (hybridization). These are the basic ingredients of the periodic Anderson Hamiltonian (PAH), which extends the one-impurity Anderson model ${ }^{3}$ to the "one-impurity-per-site" description and is one of the most studied models to describe this kind of system. We write the Hamiltonian as

$$
H=H_{a}+H_{h},
$$

separating the atomic part

$H_{a}=\sum_{i, \sigma} E n_{i \sigma}^{f}+\frac{U}{2} \sum_{i, \sigma} n_{i \sigma}^{f} n_{i \bar{\sigma}}^{f}+V \sum_{i, \sigma}\left(c_{i \sigma}^{\dagger} f_{i \sigma}+\right.$ H.c. $)$,

and the hopping term

$$
H_{h}=\sum_{i, j, \sigma} t_{i j} c_{i \sigma}^{\dagger} c_{j \sigma} \text {. }
$$

In Eqs. (2) and (3), $c_{i}^{\dagger}\left(f_{i}^{\dagger}\right)$ creates a conduction $(f)$ electron at site $i, E$ is the energy of the $f$ level, $U$ is the Coulomb repulsion between $f$ electrons, $V$ is the hybridization between $f$ and $c$ electrons (here considered as purely local), and $t_{i j}$ is the hopping matrix, or Fourier transform of the electronic kinetic energy of the conduction electrons, $\varepsilon_{\mathrm{k}}$.

Different theoretical treatments of the PAH have been explored: Green's-function techniques, variational calculations, slave boson approach, etc. Comprehensive reviews of methods and results can be found in the literature (see, for example, Ref. 4). In particular, it is illuminating to solve the atomic part, $H_{a}$, of the Hamiltonian, Eq. (2), which can be exactly diagonalized. ${ }^{5}$ Several authors have calculated physical properties in the atomic limit such as magnetic susceptibility and specific heat, ${ }^{6-8}$ and the results obtained reproduce important features observed in real systems. This fact strongly suggests an interesting approach to the problem: to start with the atomic solution and introduce the hopping term as a perturbation. The only drawback of this method resides in the complex nature of the perturbative expansion due to the presence of the interaction term in the unperturbed Hamiltonian, which prevents the applicability of Wick's theorem (see Sec. II).

Within the perturbative scheme referred to above, a simple approximation, which essentially imposes Wick's theorem, has been employed with reasonable results in the calculation of the one-particle density of states. ${ }^{5,9}$ In this paper, we use a similar approximation to calculate the static magnetic susceptibility (Sec. III) and the specific heat (Sec. IV) of the Anderson lattice in both Kondo and IV limits. The results can be successfully compared with experiments as well as other theoretical approaches. For completeness, we include in Sec. II a brief review of the method and calculation of the density of states. A final critical discussion of our results is presented in Sec. V.

\section{PERTURbation AROUND THE ATOMIC LIMIT}

As stated in Sec. I, the atomic part of the PAH, Eq. (2), can be exactly diagonalized. The energy eigenvalues $\left(E_{m}\right)$ and eigenvectors $\left(\left|\psi_{m}\right\rangle\right)$, with the corresponding 
number of particles $\left(N_{m}\right)$ and total spin $\left(S_{m}^{z}\right)$, are indicated in Table I.

We are interested in the finite-temperature one-particle Green's functions ${ }^{10}$

$$
g_{i j \sigma}^{\alpha \delta}\left(\tau_{1}-\tau_{2}\right)=-\left\langle\mathcal{T}_{\tau} a_{i \sigma}^{\alpha}\left(\tau_{1}\right) a_{j \sigma}^{\delta^{\dagger}}\left(\tau_{2}\right)\right\rangle,
$$

where the superscripts $\alpha$ and $\delta$ represent either $f$-or $c$ electron indices (e.g., $a^{f} \equiv f, a^{c^{\dagger}} \equiv c^{\dagger}$ ), $\mathcal{T}_{\tau}$ is the "time" ordering operator, and the imaginary time $\tau$ varies from zero to $\beta \equiv 1 / k_{B} T$.
From the knowledge of the eigenvalues and eigenvectors of $H_{a}$, the atomic Green's functions can be calculated, using a spectral representation ${ }^{11}$ (in the grandcanonical ensemble), yielding

$$
\begin{gathered}
g_{i \sigma}^{\alpha \delta}\left(\omega_{k}\right)=\frac{1}{Z} \sum_{m, n}\left\langle m\left|a_{i \sigma}^{\alpha}\right| n\right\rangle\left\langle n\left|a_{i \sigma}^{\delta \dagger}\right| m\right\rangle \\
\times \frac{e^{-\beta E_{m}^{\prime}}+e^{-\beta E_{n}^{\prime}}}{\omega_{m n}-i \omega_{k}},
\end{gathered}
$$

TABLE I. Number of particles, eigenvectors, eigenvalues, and spin of $H_{0}$.

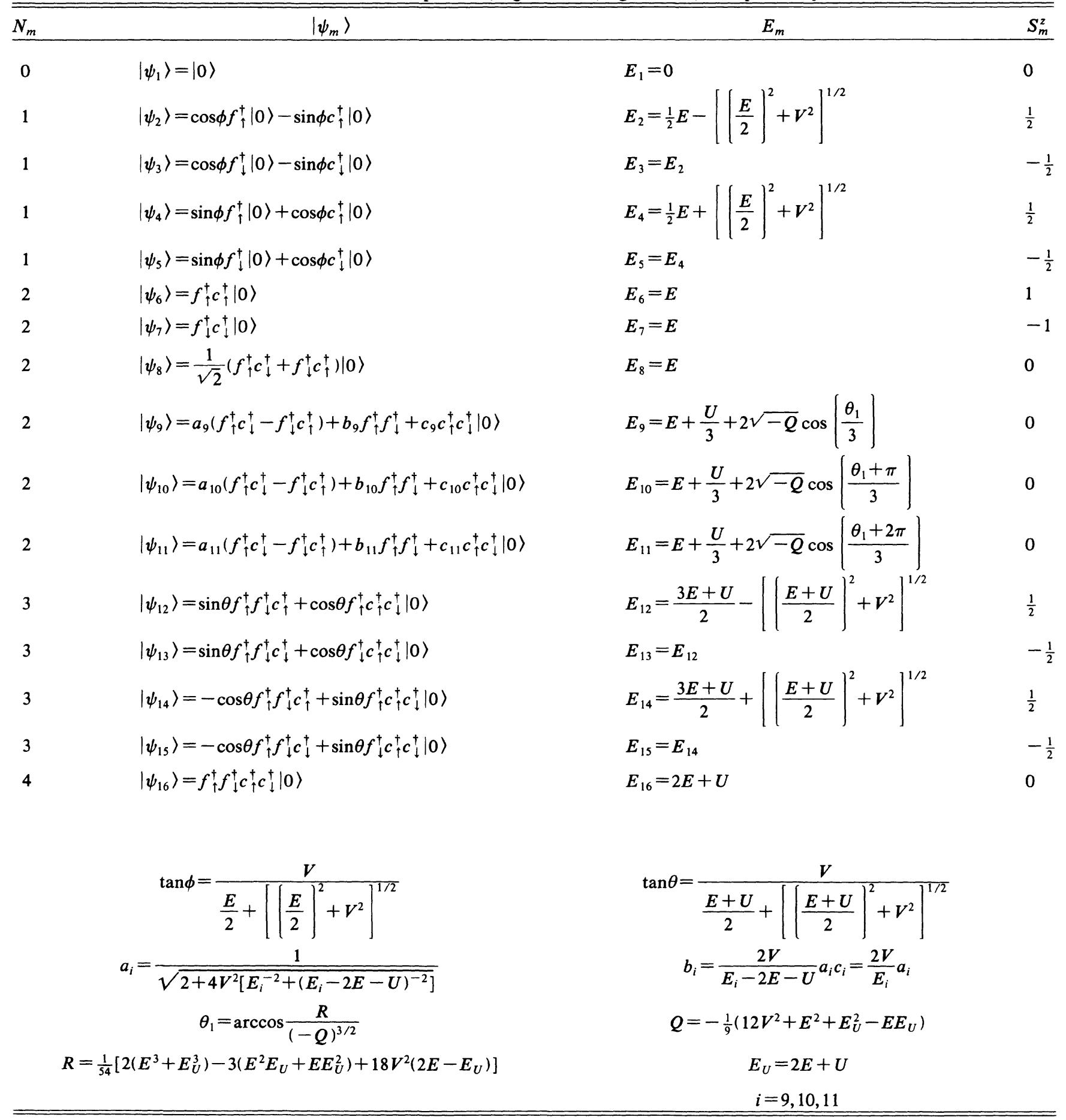


where $Z$ is the grand-canonical partition function, $\omega_{m n}=E_{m}^{\prime}-E_{n}^{\prime}, E_{m}^{\prime}=E_{m}-\mu N_{m}$, and $\omega_{k}=(\pi / \beta)(2 k+1)$, $k$ integer.

In order to calculate the full Green's function, the usual $S$-matrix perturbation expansion ${ }^{12}$ is applied to the hopping term, and the Green's functions are written as

$$
g_{i j \sigma}^{\alpha \delta}\left(\tau_{1}-\tau_{2}\right)=-\frac{\left\langle\mathcal{T}_{\tau} a_{i \sigma}^{\alpha}\left(\tau_{1}\right) a_{j \sigma}^{\delta^{\dagger}}\left(\tau_{2}\right) S(\beta)\right\rangle_{0}}{\langle S(\beta)\rangle_{0}},
$$

where

$$
\begin{aligned}
S(\beta)=\sum_{n=0}^{\infty} & \frac{(-1)^{n}}{n !} \\
& \times \int_{0}^{\beta} d \tau_{1} \cdots \int_{0}^{\beta} d \tau_{n} \mathcal{T}_{\tau} H_{h}\left(\tau_{1}\right) \cdots H_{h}\left(\tau_{n}\right) .
\end{aligned}
$$

Due to the local character of the unperturbed Hamiltonian the averages of products of fermion operators can be exactly decoupled for the different sites, e.g. (dropping the spin index),

$\left\langle\mathcal{T}_{\tau} a_{i}^{\alpha}\left(\tau_{1}\right) a_{i}^{\delta}\left(\tau_{2}\right) a_{j}^{\gamma}\left(\tau_{3}\right) \cdots a_{i}^{\gamma^{\dagger}}\left(\tau_{1}^{\prime}\right) a_{i}^{\alpha^{\dagger}}\left(\tau_{2}^{\prime}\right) a_{j}^{\delta^{\dagger}}\left(\tau_{3}^{\prime}\right)\right\rangle_{0}=\left\langle\mathcal{T}_{\tau} a_{i}^{\alpha}\left(\tau_{1}\right) a_{i}^{\delta}\left(\tau_{2}\right) a_{i}^{\gamma^{\dagger}}\left(\tau_{1}^{\prime}\right) a_{i}^{\alpha \dagger}\left(\tau_{2}^{\prime}\right)\right\rangle_{0} \cdots\left\langle\mathcal{T}_{\tau^{\prime}} a_{j}^{\gamma}\left(\tau_{3}\right) a_{j}^{\delta^{\dagger}}\left(\tau_{3}^{\prime}\right)\right\rangle_{0}$

An identical expansion, applied to the Hubbard model, has been performed formally by Metzner in a recent paper. ${ }^{13}$ We are not going to discuss the details of the perturbation expansion. Instead, we will introduce the simplest approximation, which consists in decoupling the remaining local averages of four or more fermion operators in products of pair averages. This is equivalent to imposing Wick's theorem, which is not valid due to the presence of the Coulomb interaction term in $H_{a}$. Within this approximation, the total Green's function satisfies the Dyson-like equation

$$
g_{\sigma}^{\alpha \delta}\left(\mathbf{k}, \omega_{n}\right)=g_{\sigma}^{\alpha \delta}\left(\omega_{n}\right)+g_{\sigma}^{\alpha c}\left(\omega_{n}\right) \varepsilon(\mathbf{k}) \Theta_{\sigma}^{c \delta}\left(\mathbf{k}, \omega_{n}\right)
$$

where

$$
\begin{aligned}
& \mathcal{G}_{\sigma}\left(\mathbf{k}, \omega_{n}\right)=\sum_{j} \int_{0}^{\beta} d \tau \mathcal{G}_{i j \sigma}(\tau) e^{i\left(\omega_{n} \tau-\mathbf{k} \cdot \mathbf{R}_{i j}\right)}, \\
& g_{\sigma}\left(\omega_{n}\right)=\int_{0}^{\beta} d \tau g_{\sigma}(\tau) e^{i\left(\omega_{n} \tau\right)} .
\end{aligned}
$$

We want to point out that this approximation, besides reproducing exactly the atomic limit, is also exact for zero Coulomb repulsion when Wick's theorem does apply and/or zero hybridization when the bands decouple into a purely local $f$ "band" and an uncorrelated conduction band. We also stress the fact that the important local couplings $U$ and $V$ are not treated perturbatively.

The density of states for electrons of type $\alpha$ and spin $\sigma$ is given by the relation

$$
\rho_{\sigma}^{\alpha}(\omega)=-\frac{1}{\pi} \lim _{\eta \rightarrow 0+} g_{i i \sigma}^{\alpha \alpha}(\omega+i \eta)
$$

where $g_{i i \sigma}^{\alpha \alpha}(\omega+i \eta)$ is obtained through the analytic continuation $i \omega_{n} \rightarrow \omega+i \eta$, as the retarded version of

$$
\mathcal{S}_{i i \sigma}^{\alpha \delta}\left(\omega_{n}\right) \equiv \mathcal{S}_{\sigma}^{\alpha \delta}\left(\omega_{n}\right)=\frac{1}{N} \sum_{\mathbf{k}} \mathcal{G}^{\alpha \delta}\left(\mathbf{k}, \omega_{n}\right),
$$

which is the local Green's function renormalized to include the effect of the band.

Equation (9) acquires a simple form in the case $\alpha=\delta=c$, when we have

$$
g_{\sigma}^{c c}\left(\mathbf{k}, \omega_{n}\right)=\frac{1}{\xi_{\sigma}-\varepsilon(\mathbf{k})},
$$

where

$$
\xi_{\sigma}=\xi_{\sigma}\left(\omega_{n}\right) \equiv \frac{1}{g_{\sigma}^{c c}\left(\omega_{n}\right)},
$$

which is actually spin independent.

Introducing the density of states for the uncorrelated conduction band,

$$
\rho^{0}(E)=\frac{1}{N} \sum_{\mathbf{k}} \delta(E-\varepsilon(\mathbf{k}))
$$

we can recast Eq. (9) in the form

$$
g_{\sigma}^{c c}\left(\omega_{n}\right)=\int d E \frac{\rho^{0}(E)}{\xi-E} .
$$

For simplicity, instead of calculating $\rho^{0}(E)$ for a given lattice, with a specified set of $t_{i j}$ 's, we introduce a model density of states

$$
\rho^{0}(E)=\left\{\begin{array}{l}
\left.\frac{3}{4 W} \mid 1-\frac{E^{2}}{W^{2}}\right) \quad|E| \leq W \\
0|E| \geq W .
\end{array}\right.
$$

$W$ being half the bandwidth. With this, the integral in [Eq. (15)] leads to

$g_{\sigma}^{c c}\left(\omega_{n}\right)=\frac{3}{4 W}\left[2 \xi W+\left(W^{2}-\xi^{2}\right) \ln \left(\frac{\xi+W}{\xi-W}\right)\right]$

and for the remaining Green's functions, Eq. (9) yields

$$
\begin{aligned}
& \mathcal{G}_{\sigma}^{c f}\left(\omega_{n}\right)=g_{\sigma}^{c f} \xi_{\sigma}\left(\omega_{n}\right) \mathcal{S}_{\sigma}^{c c}\left(\omega_{n}\right)=\mathcal{S}_{\sigma}^{f c}\left(\omega_{n}\right), \\
& \mathcal{G}_{\sigma}^{f f}\left(\omega_{n}\right)=g_{\sigma}^{f f}\left(\omega_{n}\right)+g_{\sigma}^{f c}\left(\omega_{n}\right) \\
& \times g_{\sigma}^{c f}\left(\omega_{n}\right) \xi\left(\omega_{n}\right) \mathcal{G}_{\sigma}^{c c}\left(\omega_{n}\right) .
\end{aligned}
$$

We used Eqs. (12), (13), (18), and (19) to obtain the $f$ and $c$ densities of states. Figure 1 shows the results at low temperatures $(T / W=0.002)$, in the symmetric case, $2 E+U=0$. The central pair of peaks corresponds to the Kondo resonance, with a gap caused by the coherent hybridization. For higher temperatures other states are excited, leading to a more complicated structure. Figure 2 shows the evolution of the density of states near the Fermi level as a function of the temperature in the nonsymmetric case $E / W=-2, U / W=6, V / W=0.1$. As in 


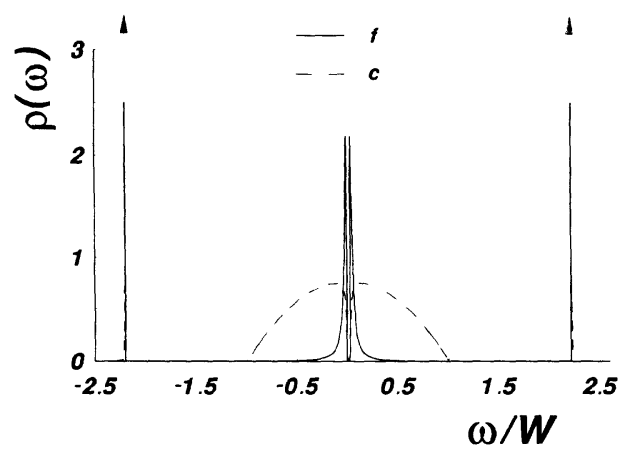

FIG. 1. Density of states at low temperatures in the symmetric case. The continuous line represents $f$ electrons, the dashed line represents $c$ electrons. The chemical potential $\mu$ lies at zero. The parameters are $T / W=0.002, E / W=-2$, $U / W=4$, and $V / W=0.3$.

the symmetric case, at low temperatures, there is a gap and two Kondo peaks near the Fermi level, but now the Fermi level is not in the gap. The peaks that appear when the temperature increases (on the left of the central gap for $T / W=0.01$ and on both sides of it for $T \geq 0.02$ ) arise from transitions between excited atomic states. The area under the Kondo peaks decreases with increasing temperature, but they do not disappear as in the impurity case. ${ }^{14}$ This is also suggested in the calculation presented by Brandow ${ }^{15}$ and in the analysis of the optical reflectivity by Marabelli, Wachter, and Walker. ${ }^{16}$

\section{STATIC MAGNETIC SUSCEPTIBILITY}

We are interested in the zero-field static susceptibility,

$$
\chi=-\left.\frac{g \mu_{B}}{2} \frac{d}{d h}\left\langle n_{\uparrow}-n_{\downarrow}\right\rangle\right|_{h=0},
$$

where

$$
n_{i \uparrow}=n_{i \uparrow}^{f}+n_{i \uparrow}^{c}
$$

In order to evaluate the magnetic susceptibility we calculated numerically Eq. (20) by adding a small external-field

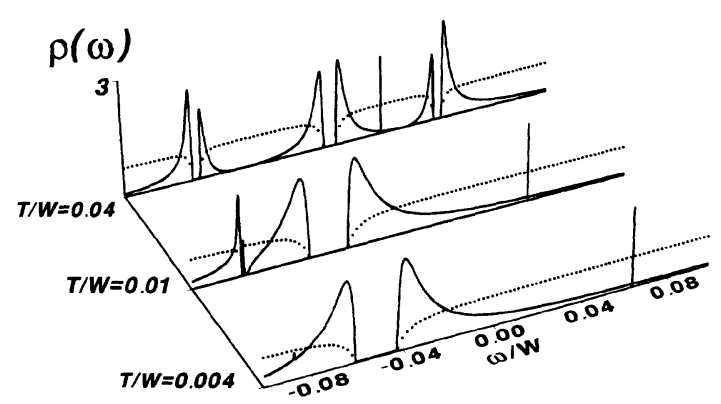

FIG. 2. View of the density of states near the Fermi level for different temperatures in the nonsymmetric case $E / W=-2$, $U / W=6$, and $V / W=0.3$. The continuous (dotted) line represents the $f$ (conduction) electrons. The vertical line stands for the chemical potential $\mu$. term to the model Hamiltonian, ${ }^{17}$

$$
H_{a}^{h}=H_{a}+g \mu_{B} h \sum_{i} S_{i z},
$$

where $g$ is the Lande factor, $\mu_{B}$ is the Bohr magneton, and the spin operator $S_{i z}$ is related to the site occupation by

$$
S_{i z}=\frac{1}{2}\left(n_{i \uparrow}-n_{i \downarrow}\right) .
$$

The number of electrons is obtained from the Green's functions through

$$
\left\langle n_{i \sigma}^{\alpha}\right\rangle=T \lim _{\tau \rightarrow 0^{-}} \sum_{\alpha, \omega_{n}} g_{i \sigma}^{\alpha \alpha}\left(\omega_{n}\right) e^{-i \omega_{n} \tau} .
$$

Notice that now the Green's function does depend on spin. From Eqs. (20) and (24) we have

$\chi_{i}=-T \lim _{\tau \rightarrow 0^{-}}\left(\frac{d}{d h} \sum_{\alpha, \omega_{n}}\left\{g_{i \uparrow}^{f f}-g_{i \downarrow}^{f f}+g_{i \uparrow}^{c c}-g_{i \downarrow}^{c c}\right\} e^{-i \omega_{n} \tau}\right)$.

The derivative with respect to the applied field in this last equation has been performed numerically.

Figure 3 shows results in the nonsymmetric Kondo case $(E / W=-2, U / W=6)$ for different hybridization values. A Curie-Weiss susceptibility is obtained for high temperatures and a Pauli-like one for low temperatures (in the symmetric case the Fermi level lies in the gap and $\chi \rightarrow 0$ for $T \rightarrow 0$ ). The itinerant nature of the lowtemperature susceptibility is confirmed by the vanishing of the local contribution (short-dashed line in Fig. 3) due to the complete suppression of the local magnetic moment (Kondo-compensated state). This is in qualitative agreement with experimental observations for certain $\mathrm{Ce}$ compounds such as $\mathrm{CeAl}_{3}, \mathrm{CeCu}_{6}$, and $\mathrm{CeCu}_{2} \mathrm{Si}_{2} \cdot{ }^{18,2}$

In the IV limit the susceptibility (Fig. 4) decreases slowly with $T$ for a large temperature range. The peak is lower than in the Kondo case and decreases for increasing hybridization (we use $E=-0.1$ and $U=2.0$ in Fig.

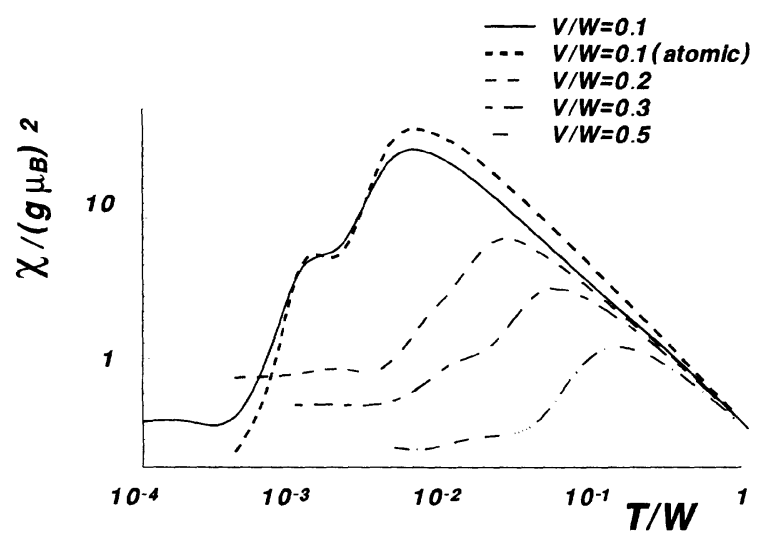

FIG. 3. Logarithm of the static susceptibility vs temperature for different values of the hybridizations $V / W=0.1$, $V / W=0.2, V / W=0.3$, and $V / W=0.5$ in the Kondo limit. The other parameters are $E / W=-2$ and $U / W=6$. 


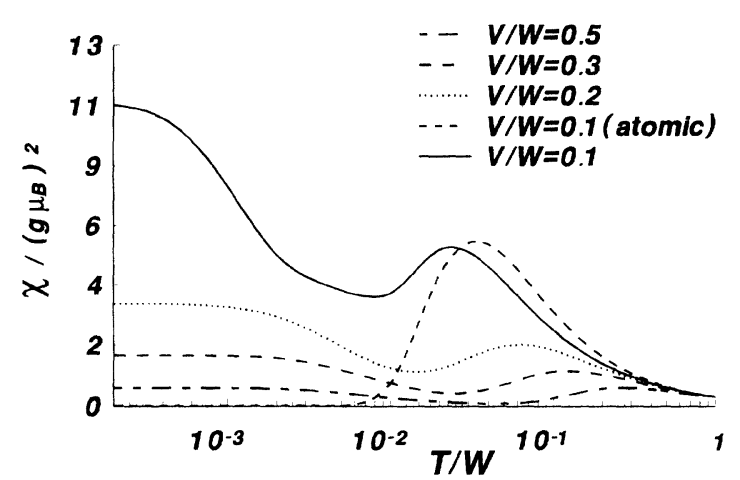

FIG. 4. Static susceptibility vs temperature in the IV case for different hybridizations $V / W=0.1, V / W=0.2, V / W=0.3$, and $V / W=0.5$. The short-dashed line is representative of the atomic case for $V / W=0.1$, the remaining plots include hopping. The other parameters are $E / W=-0.1$ and $U / W=2$.

4). There are no qualitative changes for higher $U$ values. This temperature behavior (up to $T / W \sim 0.01$ ) can be qualitatively compared with experimental results for $\mathrm{Sm}$ compounds such as $\mathrm{SmS}, \mathrm{SmSe}$, and SmTe. ${ }^{19}$ We do not expect to observe the high-temperature peak that appears in Fig. 4 in real systems; if it does appear, it should be much broader so that the susceptibility would decrease less in the region between the peaks. In fact, in our calculation, this (high-temperature) peak arises from the exponential suppression of the spin $\frac{1}{2}$ of the one- and threeparticle states which is overestimated by the local approach.

\section{SPECIFIC HEAT}

The electronic specific heat of the system is obtained as the derivative of the average energy with respect to temperature, i.e., $C=d\langle H\rangle / d T$ or, using Eqs. (1)-(3),

$$
\begin{gathered}
C=\frac{d}{d T}\left\{E\left\langle n_{\uparrow}^{f}+n_{\downarrow}^{f}\right\rangle+V\left\langle c_{\uparrow}^{\dagger} f_{\uparrow}+f_{\uparrow}^{\dagger} c_{\uparrow}+c_{\downarrow}^{\dagger} f_{\downarrow}+f_{\downarrow}^{\dagger} c_{\downarrow}\right\rangle\right. \\
\left.+U\left\langle n_{\uparrow}^{f} n_{\downarrow}^{f}\right\rangle+\left\langle H_{c}\right\rangle\right\},
\end{gathered}
$$

with

$$
\left\langle H_{c}\right\rangle=\sum_{\mathbf{k}, \sigma} \varepsilon(\mathbf{k})\left\langle c_{\mathbf{k} \sigma}^{\dagger} c_{\mathbf{k} \sigma}\right\rangle .
$$

The temperature derivative with respect to the terms on $V, E$, and $U$ in Eq. (26) has been evaluated numerically. The averages involving two operators have been obtained from the related Green's functions:

$$
\left\langle a_{\sigma}^{\delta \dagger} a_{\sigma}^{\alpha}\right\rangle=\lim _{\tau \rightarrow 0^{-}}\left[T \sum_{\omega_{n}} g_{\sigma}^{\alpha \delta}\left(\omega_{n}\right) e^{-i \omega_{n} \tau}\right] .
$$

Similarly, the fourth term of expression (26) can also be written as

$$
\left\langle H_{c}\right\rangle=\lim _{\tau \rightarrow 0^{-}}\left(T \sum_{\mathbf{k}, \omega_{n}} \varepsilon(\mathbf{k}) g^{c c}\left(\mathbf{k}, \omega_{n}\right) e^{i \omega_{n} \tau}\right) .
$$

In order to avoid a naive decoupling of the Coulomb term, we introduce another Green's function

$$
\Lambda_{i j \sigma \sigma^{\prime}}^{\alpha \beta \gamma}(\tau) \equiv\left\langle\mathcal{T}_{\tau} a_{i \sigma}^{\alpha^{\dagger}}(\tau) a_{i \sigma}^{\alpha}(\tau) a_{i \sigma^{\prime}}^{\beta}(\tau) a_{j \sigma^{\prime}}^{\gamma^{\dagger}}(0)\right\rangle,
$$

and write

$$
\left\langle n_{\sigma^{\prime}}^{\alpha} a_{\sigma^{\prime}}^{\gamma^{\dagger}} a_{\sigma^{\prime}}^{\beta}\right\rangle=\lim _{\tau \rightarrow 0^{-}}\left[T \sum_{\omega_{n}} \Lambda_{\sigma \sigma^{\prime}}^{\alpha \beta \gamma}\left(\omega_{n}\right) e^{-i \omega_{n} \tau}\right] .
$$

Following a procedure analogous to the one employed for the one-particle Green's function, we find

$$
\Lambda_{\sigma \sigma^{\prime}}^{\alpha \beta \gamma}\left(\mathbf{k}, \omega_{n}\right)=\Lambda_{(0) \sigma \sigma^{\prime}}^{\alpha \beta \gamma}\left(\omega_{n}\right)+\Lambda_{(0) \sigma \sigma^{\prime}}^{\alpha \beta c}\left(\omega_{n}\right) \varepsilon_{\sigma^{\prime}}(\mathbf{k}) \mathcal{G}_{\sigma^{\prime}}^{c \gamma}\left(\mathbf{k}, \omega_{n}\right),
$$

where $\Lambda_{(0) \sigma \sigma^{\prime}}^{\alpha \beta \gamma}\left(\omega_{n}\right)$ is the local contribution to the Green's function described by Eq. (30), which can be exactly calculated by means of the eigenstates of $H_{a}$.

The relevant $\Lambda$ Green's functions satisfy the relations

$$
\begin{aligned}
& \Lambda_{\sigma \sigma^{\prime}}^{\alpha c c}\left(\omega_{n}\right)=\Lambda_{0 \sigma \sigma^{\prime}}^{\alpha c c}\left(\omega_{n}\right)+\Lambda_{0 \sigma \sigma^{\prime}}^{\alpha c c}\left(\omega_{n}\right) H(\xi), \\
& \Lambda_{\sigma \sigma^{\prime}}^{\alpha f c}\left(\omega_{n}\right)=\Lambda_{0 \sigma \sigma^{\prime}}^{\alpha f c}\left(\omega_{n}\right)+\Lambda_{0 \sigma \sigma^{\prime}}^{\alpha f c}\left(\omega_{n}\right) H(\xi), \\
& \Lambda_{\sigma \sigma^{\prime}}^{\alpha c f}\left(\omega_{n}\right)=\Lambda_{\sigma \sigma \sigma^{\prime}}^{\alpha c f}\left(\omega_{n}\right)+\Lambda_{0 \sigma \sigma^{\prime}}^{\alpha c c}\left(\omega_{n}\right) g_{\sigma^{\prime}}^{c f} \xi\left(\omega_{n}\right) H(\xi), \\
& \Lambda_{\sigma \sigma^{\prime}}^{\alpha f f}\left(\omega_{n}\right)=\Lambda_{0 \sigma \sigma^{\prime}}^{\alpha f f}\left(\omega_{n}\right)+\Lambda_{0 \sigma \sigma^{\prime}}^{\alpha f c}\left(\omega_{n}\right) g_{\sigma^{\prime}}^{c f} \xi\left(\omega_{n}\right) H(\xi),
\end{aligned}
$$

with

$$
H(\xi)=\frac{1}{N} \sum_{\mathbf{k}} \frac{\varepsilon(\mathbf{k})}{\xi-\varepsilon(\mathbf{k})}=\xi \mathcal{G}_{\sigma}^{c c}\left(\omega_{n}\right)-1 .
$$

Figure 5 shows the results in the symmetric Kondo case with hybridizations $V=0.1$ and 0.05 . As usual, we plot the linear coefficient of the electronic specific heat, $\gamma=C / T$. In the long-dashed-line plot $(V=0.1$, including hopping) two peaks are observed: The highertemperature one is also obtained in the local (no hopping) limit (short-dashed line in Fig. 5), and can be associated with the formation of the Kondo singlet; the lowertemperature one is due to the high density of states in the vicinity of the Fermi level. The same structure can be observed for $V=0.05$ (continuous line in the same figure),

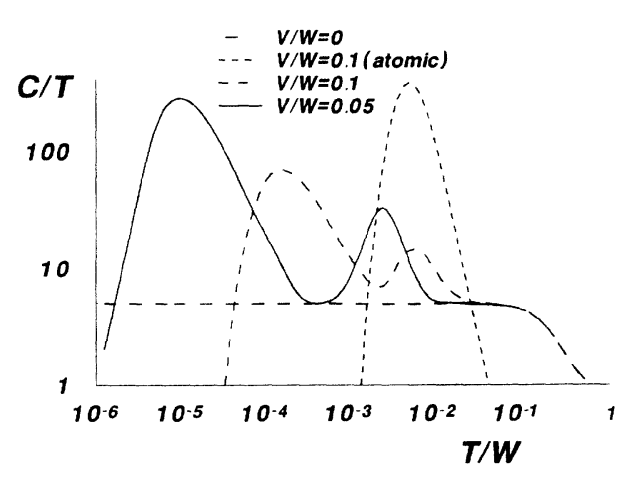

FIG. 5. Ratio of specific heat and temperature vs temperature in the Kondo limit for $V / W=0.05$ and $V / W=0.1$. The short-dashed line is representative of the atomic case for $V / W=0.1$, the remaining plots include hopping. The parameters are $E / W=-2$ and $U / W=4$. 


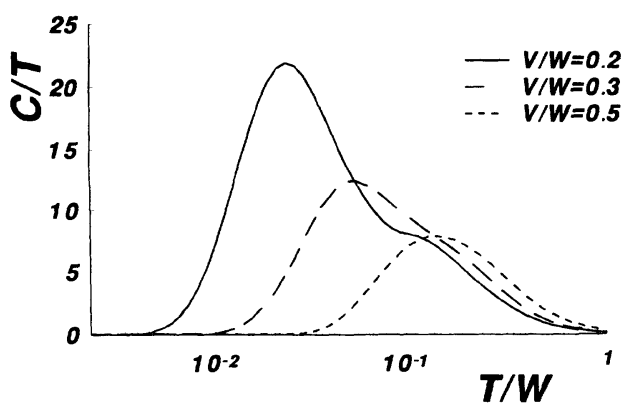

FIG. 6. Ratio of specific heat and temperature vs temperature in the IV limit for $V / W=0.2, V / W=0.3$, and $V / W=0.5$. The parameters are $E / W=-0.1$ and $U / W=2$.

the effective mass for this hybridization is $m^{*} \simeq 75$. The ratio between the amplitudes of these peaks depends on the hybridization and varies from $\sim 2$ to $\sim 10$ for $V$ ranging from 0.2 to 0.05 (with $E=-2$ ). This kind of structure has recently been observed in $\mathrm{CeCu}_{4} \mathrm{Al}$ and $\mathrm{CeCu}_{4} \mathrm{Ga},{ }^{20}$ although the high-temperature peak has been interpreted as a crystal-field effect. A similar profile, with less pronounced peaks, is also visible in a $C / T$ vs $T$ plot $^{2}$ for $\mathrm{UBe}_{13}$. We should mention the variational treatment made by Brandow, ${ }^{21}$ in which these two peaks are also found and attributed to the same origin.

Contrary to the Kondo limit, in the IV case (Fig. 6) a single peak is observed in the plot $C / T$ vs $T$ for a relevant temperature range (i.e., $T \leq 0.1$ ). This peak is a consequence of the high density of states near the Fermi level which also occurs in the IV case. Despite being lower than in the Kondo case, it explains the ratio $C / T$ observed in IV $\mathrm{Yb}$ compounds in which it reaches the value of $260 \mathrm{~mJ} / \mathrm{mol} \mathrm{K}^{2}$. $^{2}$

\section{CONCLUSIONS}

We have presented a perturbative method for approaching the Anderson lattice in which the hybridization and the Coulomb repulsion are calculated exactly in a local Hamiltonian $H_{a}$ and the hopping term is taken as a perturbation. Despite the simple approximation scheme utilized, we have found results for the density of states, for the magnetic susceptibility, and for the ratio $C / T$ in relatively good agreement with experiment. To compare with other author's results, we mention the work by Varma and Yafet. ${ }^{22}$ Using a Hubbard decoupling and a nonlocal hybridization, they find a spin susceptibility for the IV case qualitatively similar to our Kondo-limit results. Czycholl and Leder ${ }^{23}$ treat the IV case using the alloy analog approximation and find a $\mathrm{Cu}$ rie behavior for high temperatures, a maximum at some temperatures, and a finite value of the susceptibility for $T \rightarrow 0$. For the specific heat they find a large ratio $C / T$ at low temperatures, results quite similar to ours, although they calculate for an average of three particles per site. Brandow ${ }^{21}$ presents similar results for both the specific heat and the susceptibility. We also mention the results in the atomic limit presented by Alascio, Allub, and Aligia $^{6}$ and by Noce and Romano, ${ }^{7}$ to which ours reduce in the case of zero bandwidth. We should add that in our calculation of the susceptibility we considered the contribution of both $f$ and $c$ electrons, so that our zero-temperature atomic susceptibility, contrary to the above cited works, ${ }^{6,7}$ goes to zero for $T \rightarrow 0$ in the case of two particles per site. We want to stress that this approach can be applied to either the IV case or the Kondo case and, furthermore, it is worth noting that many of the characteristic features of these results are essentially due to the prominent role played by local interactions in our approach.

\section{ACKNOWLEDGMENTS}

We acknowledge fruitful discussions with B. Coqblin and C. Lacroix. This work was partially supported by Brazilian agencies Conselho Nacional de Desenvolvimento Científico e Tecnológico (CNPq), Financiadora de Estudos e Projetos (FINEP), and Fundação de Amparo à Pesquisa do Estado do Rio Grande do Sul (FAPERGS).
${ }^{1}$ See, for example, B. Coqblin, in Magnetism of Metals and Alloys, edited by M. Cyrot (North-Holland, Amsterdam, 1982), p. 295; M. B. Brandt and V. V. Moshchalkov, Adv. Phys. 33, 373 (1984).

${ }^{2}$ G. R. Stewart, Rev. Mod. Phys. 56, 755 (1984).

${ }^{3}$ P. W. Anderson, Phys. Rev. 124, 41 (1961).

${ }^{4}$ P. Fulde, J. Phys. F 18, 601 (1988).

${ }^{5}$ L. G. Brunet, R. M. Ribeiro-Teixeira, and J. R. Iglesias, Solid State Commun. 68, 477 (1988).

${ }^{6}$ B. R. Alascio, R. Allub, and A. Aligia, J. Phys. C 13, 2869 (1980).

${ }^{7}$ C. Noce and A. Romano, Physica B 160, 304 (1990).

${ }^{8}$ L. G. Brunnet, J. R. Iglesias, R. Ribeiro-Teixeira, A. R. Simðes, M. A. Gusmão, and B. Coqblin, J. Magn. Magn. Mater. 76\&77, 396 (1988).

${ }^{9}$ L. G. Brunet, R. M. Ribeiro-Teixeira, and J. R. Iglesias, J. Phys. (Paris) Colloq. 49, C8-697 (1988).

${ }^{10}$ T. Matsubara, Prog. Theor. Phys. 14, 351 (1955).
${ }^{11}$ D. N. Zubarev, Usp. Fiz. Nauk 71, 71 (1960) [Sov. Phys. Usp. 3, 320 (1960)].

${ }^{12}$ A. A. Abrikosov, L. P. Gorkov, and I. E. Dzyaloshinski, Methods of Quantum Field Theory in Statistical Physics (Prentice-Hall, Englewood Cliffs, NJ, 1963).

${ }^{13}$ W. Metzner, Phys. Rev. B 43, 8549 (1991).

${ }^{14}$ J. R. Iglesias, A. S. R. Simðes, R. M. Ribeiro-Teixeira, and L. G. Brunet, Physica B 163, 692 (1990).

${ }^{15}$ B. H. Brandow, Phys. Rev. B 33, 215 (1986).

${ }^{16}$ F. Marabelli, P. Wachter, and E. Walker, Phys. Rev. B 39, 1407 (1989).

${ }^{17}$ In a previous paper we have also calculated the finite temperature susceptibility using a spin-spin correlation function. See L. G. Brunet, M. A. Gusmão, R. M. Ribeiro-Teixeira, and J. R. Iglesias, Physica B 171, 289 (1991).

${ }^{18}$ K. Andres, J. E. Graebner, and H. R. Ott, Phys. Rev. Lett. 35, 1979 (1975).

${ }^{19}$ M. B. Maple and D. Wohlleben, Phys. Rev. Lett. 27, 511 
(1971).

${ }^{20}$ E. Bauer, N. Pillmayr, H. Müller, J. Kohlmann, and K. Winzer, J. Magn. Magn. Mater. 90\&91, 411 (1990).

${ }^{21}$ B. H. Brandow, Phys. Rev. B 37, 250 (1988).
${ }^{22}$ C. Varma and Y. Yafet, Phys. Rev. B 13, 2950 (1976).

${ }^{23}$ G. Czycholl and H. J. Leder, J. Magn. Magn. Mater. 13, 192 (1979); H. J. Leder and G. Czycholl, Z. Phys. B 35, 7 (1979). 